\title{
Rate of premalignant and malignant endometrial lesion in "low-risk" premenopausal women with abnormal uterine bleeding undergoing endometrial biopsy
}

\author{
Sangam Jha, MS ${ }^{1}$, Akanksha Singh, MBBS ${ }^{1}$, Hemali Heidi Sinha, $\mathrm{MS}^{1}$, Poonam Bhadani, MD², \\ Monika Anant, MS' ${ }^{1}$, Mukta Agarwal, MD'1 \\ ${ }^{1}$ Department of Obstetrics and Gynecology, ${ }^{2}$ Department of Pathology, All India Institute of Medical Science Patna, Bihar, India
}

Objective

To determine the incidence of endometrial hyperplasia and endometrial cancer (EH/EC) in low-risk premenopausal women with abnormal uterine bleeding (AUB) undergoing endometrial biopsy and to build a predictive model that includes clinical variables for predicting EH/EC in these women.

Methods

This retrospective study was conducted between January 2015 and March 2020. All premenopausal women aged $<55$ years with AUB who underwent endometrial sampling during a specified time period were included. Data regarding baseline characteristics, sonographic findings, and histological reports were collected from patient record sheets.

\section{Results}

During the specified time period, 1,089 premenopausal women underwent endometrial biopsy for AUB. Complete data analysis was done for 1,084 women. Of the endometrial samples, $95.3 \%$ revealed benign pathology, whereas $4.7 \%$ of the samples had major endometrial pathology EH/EC. On step-wise logistic regression analysis, intermenstrual bleeding (IMB) $(\mathrm{OR}, 3.15)$, body mass index $(\mathrm{BMI})>25 \mathrm{~kg} / \mathrm{m}^{2}$ (odds ratio [OR], 4.4705), age $>40$ years $(\mathrm{OR}, 1.14)$, endometrial thickness (ET) $>13 \mathrm{~mm}(\mathrm{OR}, 2.59)$, and hypothyroidism (OR, 1.35) were significantly associated with EH/ EC. Considering the pretest probability for an EH/EC of $4.7 \%$, this prediction model with a likelihood ratio of $14.2 \%$ demonstrated a post-test probability of $41 \%$ in the presence of the above-mentioned variables.

\section{Conclusion}

The risk of EH/EC was lower in low-risk premenopausal women with AUB. However, premenopausal women with IMB aged $>40$ years, hypothyroidism, BMI $>25 \mathrm{~kg} / \mathrm{m}^{2}$, and thickened endometrium (ET $>13 \mathrm{~mm}$ ) are at high risk of EH/EC; therefore, endometrial biopsy should be considered early in their management plan.

Keywords: Endometrial hyperplasia; Endometrial cancer; Biopsy; Premenopausal; Hypothyroidism

\section{Introduction}

Abnormal uterine bleeding (AUB) is defined as uterine bleeding that is abnormal in duration or timing. It affects $14-25 \%$ of women of reproductive age $[1,2]$. Most of the causes of AUB are benign; however, it is also the most common symptom of endometrial hyperplasia $(\mathrm{EH})$ and endometrial carcinoma (EC). Although the incidence of endometrial cancer increases after menopause, $10 \%$ of it occurs before menopause [3]. Atypical EH is associated with a concurrent carcinoma in up to $50 \%$ of cases [4], along with the risk of
Received: 2021.05.10. Revised: 2021.06.19. Accepted: 2021.09.07 Corresponding author: Sangam Jha, MS

Department of Obstetrics and Gynecology, All India Institute of Medical Science Patna, Aurangabad Rd, Phulwari Sharif, Patna, Bihar 801507, India

E-mail: sangam.jha78@gmail.com

https://orcid.org/0000-0002-4349-1589

Articles published in Obstet Gynecol Sci are open-access, distributed under the terms of the Creative Commons Attribution Non-Commercial License (http://creativecommons. org/licenses/by-nc/3.0/) which permits unrestricted non-commercial use, distribution, and reproduction in any medium, provided the original work is properly cited.

Copyright $\odot 2021$ Korean Society of Obstetrics and Gynecology 


\title{
Obstetrics \& Gynecology Science
}

\author{
Vol. 64, No. 6, 2021
}

neoplastic progression of $12.5 \%$ in the following 10 years [5]; hence, it is managed similarly to EC with hysterectomy. Guidance on the performance of endometrial biopsy in postmenopausal bleeding is clear, whereas evidence on its utilization in premenopausal women with AUB is inconclusive. To overcome this, many clinical variables such as nulliparity, obesity, diabetes, hypertension, tamoxifen therapy, and infertility have been considered to assess the risk of endometrial cancer in this age group. Based on these risk factors, the Society of Obstetricians and Gynecologists of Canada (SOGC) recommends endometrial biopsy in women aged over 40 years or in younger women in the presence of risk factors for endometrial cancer [6], whereas the American College of Obstetricians and Gynecologists (ACOG) recommends endometrial sampling in women aged $>45$ years or earlier in the presence of risk factors [7]. Most of these guidelines are based on studies of the Western female population. Given the racial variation between Western and Asian females, risk factors would also be different. Therefore, we need separate risk stratification for them. Although most endometrial cases are preceded by $A \cup B$, the incidence of $E C$ is only $1-2 \%$ in premenopausal women with AUB. This indicates that a large number of women do not necessarily undergo endometrial biopsy to rule out cancer. Therefore, careful selection of patients by estimating absolute risk will avoid unnecessary sampling as well as false-positive cases. In this regard, to define women at risk of EH/EC, this study aimed to estimate the risk of significant endometrial pathology and create a predictive model based on clinical variables such as age, body mass index (BMI), menstrual pattern, and transvaginal sonography findings in these populations.

\section{Materials and methods}

This retrospective cohort study was conducted after obtaining approval from the Institutional Ethical Committee (AlIMS/ Pat/IEC/2020-635) from January 2015 to March 2020 in the Department Of Obstetrics And Gynecology at the All India Institute of Medical Science, Patna. AUB was defined by symptoms such as heavy menstrual bleeding if pictorial blood assessment chart (PBAC) score $>100$ (PBAC, method quantifying menstrual blood loss by recording the number of tampons or towels used and the degree to which they are stained with blood), intermenstrual bleeding (occurring between well-defined cyclical menses), postcoital bleeding $(\mathrm{PCB})$, or irregular menses (when the cycle-to-cycle variation is $>20$ days within one 90 -day reference period).

All women aged $<55$ years who presented with AUB and had undergone endometrial biopsy during the specified time period were included in the study. Endometrial biopsy was performed by the aspiration method using Karman's cannula as an outpatient department procedure.

Post-menopausal women (amenorrhea for $>1$ year), women with a history of diabetes, hypertension, infertility, chronic anovulation, tamoxifen use, or family history of uterine, ovarian, breast, and colon cancer were excluded from the study.

Participants were identified from the laboratory database and clinical and demographic variables such as age, BMI,

Table 1. Characteristics of study population $(n=1,084)$

\begin{tabular}{|c|c|}
\hline Variables & Value \\
\hline \multicolumn{2}{|l|}{ Age (yr) } \\
\hline$\leq 40$ & $385(35.5)$ \\
\hline$>40$ & $699(64.5)$ \\
\hline \multicolumn{2}{|l|}{ Menstrual cycle phase } \\
\hline Proliferative phase & $278(25.6)$ \\
\hline Secretory phase & $542(74.4)$ \\
\hline \multicolumn{2}{|l|}{ Parity } \\
\hline$\leq 1$ & $101(9.3)$ \\
\hline$>1$ & $983(90.7)$ \\
\hline \multicolumn{2}{|l|}{ Body mass index $\left(\mathrm{kg} / \mathrm{m}^{2}\right)$} \\
\hline$\leq 25$ & $552(50.9)$ \\
\hline$>25$ & $532(49.1)$ \\
\hline Hypothyroidism & $57(5.2)$ \\
\hline Endometrial thickness (median, mm) & 9.4 \\
\hline \multicolumn{2}{|l|}{ Menstrual pattern } \\
\hline PCB & $16(1.4)$ \\
\hline $\mathrm{HMB}$ & $941(86.8)$ \\
\hline IMB & $127(11.7)$ \\
\hline Hormone use & $44(4)$ \\
\hline \multicolumn{2}{|l|}{ Histology } \\
\hline EC & $20(1.8)$ \\
\hline $\mathrm{AEH}$ & $31(2.8)$ \\
\hline Benign pathology & $1,033(95.29)$ \\
\hline
\end{tabular}

Values are presented as number (\%).

$P C B$, postcoital bleeding; $H M B$, heavy menstrual bleeding; IMB, intermenstrual bleeding; EC, endometrial carcinoma; $A E H$, atypical endometrial hyperplasia. 


\section{Obstetrics \& Gynecology Science}

Sangam Jha, et al. Risk of significant endometrial pathology in low-risk women

parity, menstrual history, medical history (smoking, diabetes, hypothyroidism, bleeding disorder, breast cancer, colorectal cancer, self-reported use of hormone therapy), infertility (>12 months), and family history (breast, colorectal, or endometrial cancer), recent investigations (hemoglobin, pelvic ultrasound scan), and histopathology report data were collected from the patient's record sheet.

Significant endometrial pathology included EH with atypia and EC. The histological diagnosis of EH with or without atypia was based on the WHO 2014 classification [8].

\section{Statistical analysis}

Qualitative data were expressed as numbers and percentages. The Kolmogorov-Smirnov test was used to test for normality. Stepwise logistic regression analysis was performed to identify variables that were significantly associated with endometrial pathology EH/EC. Stepwise logistic regression analysis was performed with an entry and exit $P$-value of 0.3/0.5. Results were expressed as odds ratio with $95 \%$ confidence intervals. The predicted probabilities of step-wise logistic analysis were used to form receiver operating characteristic (ROC) curves and to calculate sensitivity, specificity, negative predictive value, positive predictive value, and posi- tive and negative likelihood ratios. The chi-square test was used to compare the association of more than one risk factor with significant endometrial pathology and non-significant endometrial pathology. Statistical significance was set at $P<0.05$. All statistical analyses were performed using MedCalc Statistical software version 19.2.6 (MedCalc Software bvba, Ostend, Belgium).

\section{Results}

A total of 1,089 premenopausal women underwent endometrial biopsy for AUB during the specified time period. Data for five women were incomplete, so the final analysis was performed for 1,084 women. The baseline characteristics of the study population are summarized in Table 1. Of the women, $9.1 \%$ were nulliparous, and $90 \%$ were multiparous. Hypothyroidism was present in 57 women (5.2\%). The median endometrial thickness in the studied population was $9.4 \mathrm{~mm}$. Heavy menstrual bleeding was reported by 941 (86.8\%) women, whereas intermenstrual bleeding (IMB) was noted in $127(11.7 \%)$ and PCB in $16(1.4 \%)$.

Most of the endometrial samples (95.3\%) had benign pa-

Table 2. Step-wise logistic regression analysis of the variables for predicting significant endometrial pathology

\begin{tabular}{lccc}
\hline Variables & Odds ratio & $\mathbf{9 5 \% ~ C l}$ & $\boldsymbol{P}$-value \\
\hline Age (cut off at $>40$ years) & 1.14 & $0.99-1.20$ & $<0.0001$ \\
BMI (cut off at $>25 \mathrm{~kg} / \mathrm{m}^{2}$ ) & 4.40 & $1.51-14.36$ & 0.0072 \\
Endometrial thickness (cut off at $>13 \mathrm{~mm}$ ) & 2.59 & $1.78-3.77$ & 0.0001 \\
Hormone use & 0.09 & $0.004-2.11$ & 0.135 \\
Hypothyroidism & 1.35 & $0.64-1.94$ & 0.001 \\
Menstrual pattern $(\mathrm{IMB})$ & 3.15 & $1.90-5.23$ & 0.004 \\
Parity $(<1)$ & 0.03 & $0.003-0.34$ & 0.14 \\
\hline
\end{tabular}

$\mathrm{Cl}$, class interval; BMI, body mass index; IMB, intermenstrual bleeding.

Table 3. Statistics of the variables for predicting EH/EC pathology

\begin{tabular}{lcccc}
\hline Variables & Sensitivity $\mathbf{( 9 5 \% ~ C l )}$ & Specificity (95\% Cl) & LR+ (95\% Cl) & LR- (95\% Cl) \\
\hline Age $(>40$ years) & $82.35(69.1-91.6)$ & $49.66(46.6-52.8)$ & $1.64(1.4-1.9)$ & $0.36(0.2-0.6)$ \\
BMI $\left(>25 \mathrm{~kg} / \mathrm{m}^{2}\right)$ & $62.75(48.1-75.9)$ & $52.02(48.9-55.1)$ & $1.31(1.0-1.6)$ & $0.72(0.5-1.0)$ \\
Endometrial thickness $(>13 \mathrm{~mm})$ & $88.24(76.1-95.6)$ & $98.07(97.0-98.8)$ & $45.79(29.3-71.5)$ & $0.12(0.06-0.3)$ \\
Hypothyroidism & $64.71(50.1-77.6)$ & $57.30(54.1-68.2)$ & $23.99(15.8-36.4)$ & $0.36(0.3-0.5)$ \\
Menstrual pattern $(\mathrm{IMB})$ & $82.35(69.1-91.6)$ & $90.17(88.2-91.9)$ & $8.38(6.7-10.5)$ & $0.20(0.1-0.4)$ \\
\hline
\end{tabular}

EH/EC, endometrial hyperplasia and endometrial cancer; Cl, class interval; LR+, positive likelihood ratio; LR-, negative likelihood ratio; BMI, body mass index; IMB, intermenstrual bleeding. 


\title{
Obstetrics \& Gynecology Science
}

\author{
Vol. 64, No. 6, 2021
}

thology on HPE. Thirty-one (2.8\%) endometrial samples were diagnosed with EH with atypia, and twenty (1.84\%) were diagnosed with endometrial cancer on histology combined with $4.7 \%$ of significant endometrial pathology.

On step-wise logistic regression analysis, IMB (odds ratio $[O R]=3.15), B M l>25 \mathrm{~kg} / \mathrm{m}^{2}$ (OR=4.4705), and hypothyroidism $(\mathrm{OR}=1.3)$ were significantly associated with $\mathrm{EH} / \mathrm{EC}$ (Table 2). Predictive probabilities of stepwise logistic regression were used to create the ROC curve. On ROC curve analysis, the best age cut-off value was $>40$ years and endometrial thickness cut-off value was $>13 \mathrm{~mm}$ for predicting significant endometrial pathology. The variables that were not included in the predictive model were hormone use, parity, and menstrual cycle phase. Table 3 summarizes the predictive probabilities of the different variables for the EH/EC. Predictive probabilities of the logistic regression analysis were used to create a full ROC curve and calculate the sensitivity, specificity, positive and negative predictive values, and positive and negative likelihood ratios of the predictive model. The estimated AUC was 0.971 (95\% class interval, 0.959-0.980) $(P<0.0001)$ (Fig. 1). At the best cut-off value, the positive likelihood ratio was

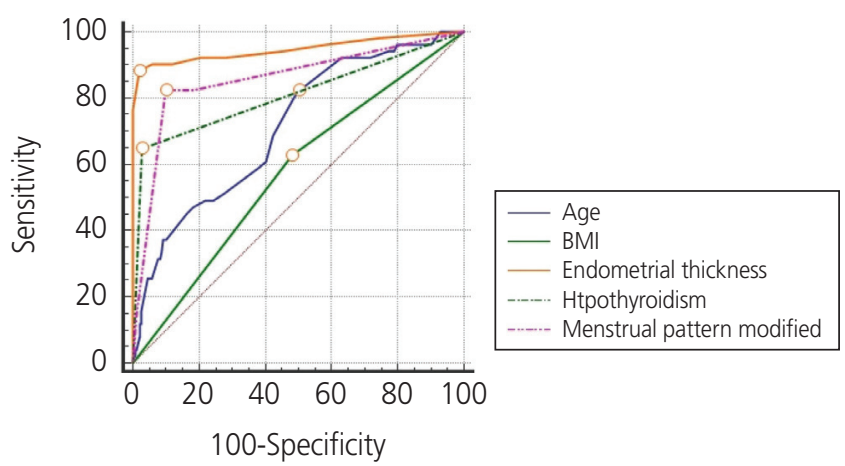

Fig. 1. Receiver operating characteristics curve of the predictive model. The estimated area under the curve was 0.971 (95\% class interval, $0.959-0.980)$ at $P<0.0001$. BMI, body mass index.
14.2 at 0.2 negative likelihood ratio resulting in a post-test probability of $41 \%$ at a pre-test probability of $4.7 \%$. The presence of one or more risk factors was more common in the EH/EC group than in the benign group (Table 4).

\section{Discussion}

Approximately $50 \%$ of women affected with AUB are in the 40-50 years age group [9]. The most common cause of AUB in this age group was benign. In the present study, benign endometrial pathology was noted in most women (95\%). The reported incidence of major endometrial pathology $(\mathrm{EH} /$ EC) in premenopausal women with AUB is wide, ranging from $5 \%$ to $14 \%[10,11]$. This might be due to differences in the studied populations (high risk and low risk). Our study suggested a $4.7 \%$ prevalence rate of significant endometrial pathology in low-risk women with AUB. This was similar to the previously reported prevalence among low-risk women $[11,12]$. Although the incidence of EC is low in these women, the risk of progression of non-atypical and atypical $\mathrm{EH}$ to endometrial cancer is $10 \%$ and $40 \%$, respectively, which is quite significant [5]. In addition, one-third of women with atypical hyperplasia have concurrent carcinoma [13]. Hence, careful patient selection to diagnose and treat significant endometrial pathology is very important.

Age is the independent variable to decide on endometrial biopsy in premenopausal women with AUB. SOGC suggests that endometrial biopsy should be performed in women aged over 40 years, whereas ACOG has set the age cut-off to 45 years [6,7]. Wise et al. [9] and Giannella et al. [11] reported no correlation between age and EC [9]. To add to this ongoing debate on age cut-off, we, on full ROC curve analysis, found $>40$ years as the best age cut-off. In a systemic review on the risk of endometrial cancer in premenopausal

Table 4. Presence of risk factors in women with and without $E H / E C$

\begin{tabular}{|c|c|c|c|}
\hline $\begin{array}{l}\text { Risk factors (age, BMI, parity, menstrual pattern } \\
\text { hypothyroidism, and thickened ET) }\end{array}$ & Women with EH/EC & Women without EH/EC & $P$-value \\
\hline 1 risk factor & 0 & $235(22.7)$ & $<0.0001$ \\
\hline 2 risk factor & $2(3.9)$ & $19(1.8)$ & $<0.0001$ \\
\hline 3 risk factor & $14(27.4)$ & $11(1.1)$ & $<0.0001$ \\
\hline$>3$ risk factor & $35(68.6)$ & 0 & $<0.0001$ \\
\hline
\end{tabular}

Values are presented as number (\%).

$\mathrm{EH} / \mathrm{EC}$, endometrial hyperplasia and endometrial cancer; BMI, body mass index; ET, endometrial thickness. 


\section{Obstetrics \& Gynecology Science}

Sangam Jha, et al. Risk of significant endometrial pathology in low-risk women

women, Pennant et al. [14] reported an increase in the risk for women aged $>40$ years compared to those aged $<40$ years.

A higher BMI has been associated with an increased risk of $\mathrm{EH}$ or cancer. A recent study from New Zealand on the association of BMl with endometrial cancer reported that obese women $\left(\mathrm{BMl}>30 \mathrm{~kg} / \mathrm{m}^{2}\right.$ ) are four times more likely to have EH/EC compared to normal weight women [9]. The Asian population has a lower mean BMI but a greater tendency toward abdominal adiposity than the non-Asian population. Furthermore, Asian women have a higher percentage of body fat at low BMl compared to age-specific non-Asian women, which also reflects the increased risk of chronic diseases (e.g., diabetes mellitus, cardiovascular, hypertension) [15]. In this study, women with $\mathrm{BMI}>25 \mathrm{~kg} / \mathrm{m}^{2}$ were 4.4 times more likely to develop EH/EC compared to women with $\mathrm{BMl}<25 \mathrm{~kg} / \mathrm{m}^{2}$. This is in accordance with previous literature $[9,11,16]$.

Recently, hypothyroidism has been linked to EC. Although the direct relationship between EC and hypothyroidism remains to be elucidated, it has been postulated to influence the disease either by interfering with risk factors such as infertility, polycystic ovary syndrome, and metabolic syndrome or by directly acting on thyroid-related receptors of the endometrium [17]. The present study showed an association between hypothyroidism and EH/EC, with an adjusted OR of 1.3 .

Endometrial thickness had reproduced greater predictability for EC in postmenopausal women, but in premenopausal women due to periodic changes in the thickness in various phases of the endometrial cycle, its predictability is poor; hence, the best cut-off value for performing endometrial biopsy is debatable. Few studies have recommended endometrial biopsy at endometrial thickness (ET) $>8 \mathrm{~mm}$ as the risk of EH/EC increases [18], whereas few studies have shown a strong association at ET >11 mm $[9,11]$. In the current study, a strong association was observed for ET $>13 \mathrm{~mm}$. In the present study, women with an IMB pattern had been shown to a 3.1 times higher risk of having EH/EC compared to other menstrual patterns. Gawron et al. [19] also reported that IMB increases the chance by 4.1 times for the diagnosis of EC. In this study, it was observed that women with significant endometrial pathology had the simultaneous presence of multiple risk factors.

The prediction model including age $>40$ years, $\mathrm{BMI}$
$>25 \mathrm{~kg} / \mathrm{m}^{2}$, hypothyroidism, and menstrual pattern showed good diagnostic accuracy with an AUC of 0.962, a positive likelihood ratio of 7.9 , and a negative likelihood ratio of 0.67 . When endometrial thickness $(>13 \mathrm{~mm}$ ) was taken into account, the positive likelihood ratio increases to 14.2 at a negative likelihood ratio of 0.2 . Considering the disease prevalence of $4.7 \%$, the presence of these risk factors would elevate the risk of the disease to $41 \%$, whereas the absence of the above-mentioned risk factors would reduce the disease risk to $2.5 \%$. The findings of this study suggest that the prevalence of significant endometrial pathology in low-risk premenopausal women with AUB is less, but simultaneously emphasizes that the presence of the above-mentioned variables puts them at higher risk of harboring the disease and hence should be considered for endometrial biopsy.

\section{Limitation and strength of the study}

The main limitation of this study is its retrospective nature; therefore, data were collected from the medical record sheet of the patients. There may be unmeasured confounders not included in the analysis, which could influence the study results. The prevalence was low; therefore, it might not represent the true prevalence of the disease. Histopathology was not reviewed for the purpose of the study, but this adds strength to the study by making the results more generalizable. The major strengths of the study are the large sample size, all clinical variables included in the study were measurable, and all patients underwent histological examination as a reference value. However, there should be a prospective study in a community setting to estimate the true incidence of significant endometrial pathology in women with AUB.

\section{Conclusion}

In low-risk premenopausal women with $A \cup B$, the presence of clinical variables such as age $>40$ years, BMl $>25 \mathrm{~kg} / \mathrm{m}^{2}$, hypothyroidism, IMB, and ET $>13 \mathrm{~mm}$ increased the risk for EH/EC. Hence, these women should be evaluated using endometrial biopsy.

\section{Conflict of interest}

No potential conflict of interest relevant to this article was reported. 


\section{Obstetrics \& Gynecology Science}

Vol. 64, No. 6, 2021

\section{Ethical approval}

Ethical approval and consent to participate - The study was approved by the Institutional Ethical Committee (AllMS/Pat/ IEC/2020/635).

\section{Patient consent}

Verbal consent was obtained from all study participants. This procedure was approved by the ethical committee.

\section{Funding information}

None.

\section{References}

1. Fraser IS, Langham S, Uhl-Hochgraeber K. Health-related quality of life and economic burden of abnormal uterine bleeding. Expert Rev Obstet Gynecol 2009;4:179-89.

2. Munro MG, Critchley HO, Broder MS, Fraser IS, FIGO Working Group on Menstrual Disorders. FIGO classification system (PALM-COEIN) for causes of abnormal uterine bleeding in nongravid women of reproductive age. Int J Gynaecol Obstet 2011;113:3-13.

3. Bignardi T, Van den Bosch T, Condous G. Abnormal uterine and post-menopausal bleeding in the acute gynaecology unit. Best Pract Res Clin Obstet Gynaecol 2009;23:595-607.

4. Lacey JV Jr, Chia VM. Endometrial hyperplasia and the risk of progression to carcinoma. Maturitas 2009;63:3944.

5. Lacey JV Jr, Sherman ME, Rush BB, Ronnett BM, loffe $\mathrm{OB}$, Duggan MA, et al. Absolute risk of endometrial carcinoma during 20-year follow-up among women with endometrial hyperplasia. J Clin Oncol 2010;28:788-92.

6. Singh S, Best C, Dunn S, Leyland N, Wolfman WL, Clinical Practice-Gynaecology Committee. Abnormal uterine bleeding in pre-menopausal women. J Obstet Gynaecol Can 2013;35:473-5.

7. Committee on Practice Bulletins-Gynecology. Practice bulletin no. 128: diagnosis of abnormal uterine bleed- ing in reproductive-aged women. Obstet Gynecol 2012;120:197-206.

8. Emons G, Beckmann MW, Schmidt D, Mallmann P, Uterus commission of the Gynecological Oncology Working Group (AGO). New WHO classification of endometrial hyperplasias. Geburtshilfe Frauenheilkd 2015;75:135-6.

9. Wise MR, Gill P, Lensen S, Thompson JM, Farquhar CM. Body mass index trumps age in decision for endometrial biopsy: cohort study of symptomatic premenopausal women. Am J Obstet Gynecol 2016;215:598.e1-8.

10. Farquhar CM, Lethaby A, Sowter M, Verry J, Baranyai J. An evaluation of risk factors for endometrial hyperplasia in premenopausal women with abnormal menstrual bleeding. Am J Obstet Gynecol 1999;181:525-9.

11. Giannella L, Cerami LB, Setti T, Bergamini E, Boselli F. Prediction of endometrial hyperplasia and cancer among premenopausal women with abnormal uterine bleeding. Biomed Res Int 2019;2019:8598152.

12. Sattanakho P, Kleebkaow P, Sangkomkumhang U, Booranabunyat S, Buppasiri P. Rate of significant endometrial pathology in women at low risk for endometrial hyperplasia or cancer presenting with abnormal uterine bleeding. Pragmat Obs Res 2020;11:13-8.

13. Doherty MT, Sanni OB, Coleman HG, Cardwell CR, MCCluggage WG, Quinn $D$, et al. Concurrent and future risk of endometrial cancer in women with endometrial hyperplasia: a systematic review and meta-analysis. PLoS One 2020;15:e0232231.

14. Pennant ME, Mehta R, Moody P, Hackett G, Prentice A, Sharp SJ, et al. Premenopausal abnormal uterine bleeding and risk of endometrial cancer. BJOG 2017;124:40411.

15. Deurenberg-Yap M, Schmidt G, van Staveren WA, Deurenberg $P$. The paradox of low body mass index and high body fat percentage among Chinese, Malays and Indians in Singapore. Int J Obes Relat Metab Disord 2000;24:1011-7.

16. Guraslan H, Dogan K, Kaya C, Senturk MB, Guraslan B, Helvacioglu $C$, et al. Could body mass index be an indicator for endometrial biopsy in premenopausal women with heavy menstrual bleeding? Arch Gynecol Obstet 2016;294:395-402.

17. Wang Y, Zhou R, Wang J. Relationship between hypothyroidism and endometrial cancer. Aging Dis 2019;10:190-6. 


\section{Obstetrics \& Gynecology Science}

Sangam Jha, et al. Risk of significant endometrial pathology in low-risk women

18. Park YR, Lee SW, Kim Y, Bae IY, Kim HK, Choe J, et al. Endometrial thickness cut-off value by transvaginal ultrasonography for screening of endometrial pathology in premenopausal and postmenopausal women. Obstet Gynecol Sci 2019;62:445-53.
19. Gawron I, Łoboda M, Babczyk D, Ludwin I, Basta P, Pityński K, et al. Endometrial cancer and hyperplasia rate in women before menopause with abnormal uterine bleeding undergoing endometrial sampling. Przegl Lek 2017;74:139-43. 